CASE REPORT

\author{
J.A. Yu \\ R. Sohaey \\ A.M. Kennedy \\ N.R. Selden
}

\section{Terminal Myelocystocele and Sacrococcygeal Teratoma: A Comparison of Fetal Ultrasound Presentation and Perinatal Risk}

SUMMARY: This case exemplifies the difficulty in differentiating cystic sacrococcygeal teratoma and terminal myelocystocele. Fetal sonography presentation and perinatal risks of sacrococcygeal teratoma and terminal myelocystocele are compared, and we emphasize the importance of obtaining fetal MR imaging to establish an accurate diagnosis.
$\mathbf{T}$ he terminal spine presents unique imaging challenges, particularly in the case of large cystic masses. In the first case, an isolated terminal myelocystocele mimicked a cystic sacrococcygeal teratoma (SCT) on prenatal sonography. The second case of type 2 (SCT) is presented for comparison. We discuss the presentation and perinatal risks of these 2 cystic sacrococcygeal masses and illustrate surgical correction of a cystic terminal myelocystocele.

\section{Case Reports}

\section{Index Case}

A 22-year-old gravida 2, para 1 was referred to us for investigation of a fetal mass. Maternal serum alpha-fetoprotein (AFP) levels were mildly elevated, and amniocentesis results were a healthy female with normal acetylcholinesterase levels.

At 30-weeks gestational age, sonography showed a cystic exophytic lower pelvic mass measuring $8 \times 7 \times 6 \mathrm{~cm}$. The mass was skin-covered and septate (Fig $1 A,-B$ ). It appeared to originate from the tip of an otherwise normal sacrum. Fetal calvaria views were normal, without Chiari II malformation. Amniotic fluid index (AFI) measured $21 \mathrm{~cm}$, and fetal size was appropriate for gestational age. The size, location, and cystic composition of the mass and the perceived absence of skeletal dysraphism and Chiari II malformation supported the diagnosis of cystic SCT with complicating polyhydramnios. Fetal MR imaging was recommended to better characterize the mass; however, the patient refused due to claustrophobia. The follow-up fetal sonography at 36 weeks showed the mass had grown $(10 \times 10 \times 6 \mathrm{~cm})$ and the AFI had increased to $24 \mathrm{~cm}$.

At 37 weeks, the neonate was delivered via classic cesarean delivery without complications, weighing $3765 \mathrm{~g}$ with Apgar scores of 2, 5, and 8 , at 1, 5, and 10 minutes, respectively. The mode of delivery was based on the prenatal diagnosis of a large SCT.

Neonatal CT and MR imaging of the congenital cyst and spine revealed sacral spinal dysraphism and tethered cord (Fig $1 C,-D)$. The diagnosis was revised to terminal myelocystocele.

Surgical exploration was undertaken at 7 days of life (Fig $1 E,-F)$. The large cyst was entered and found to contain clear CSF in a termi-

Received August 25, 2006; accepted after revision October 1.

From the School of Medicine (J.A.Y.) and the Departments of Diagnostic Radiology (R.S.) and Neurological Surgery (N.R.S.), Oregon Health \& Science University, Portland, Ore; and the Department of Radiology (A.M.K.), University of Utah, College of Medicine, Salt Lake City, Utah.

Please address correspondence to Nathan R. Selden, MD, PhD, FACS, FAAP, Department of Neurological Surgery, CH 8N, Oregon Health \& Science University, 3303 S.W. Bond Ave., Portland, OR 97239; e-mail: seldenn@ohsu.edu

DOI 10.3174/ajnr.A0502 nal continuation of the spinal subarachnoid space. A second thinwalled cyst encountered at the base of the larger cyst represented terminal dilation of the spinal cord central canal (Fig 1G). These findings are diagnostic of terminal myelocystocele. ${ }^{1}$ Opening the second cyst revealed the true spinal canal, with lipomatous distal tethering of the cord to the dorsal and cranial aspect of the myelocystocele (Fig $1 H$ ). Primary spinal cord untethering was undertaken, and the spinal canal was closed by using duraplasty material. Postoperatively, the neonate retained normal neurologic and urologic function.

\section{Comparison Case}

The images from another fetus with type 2 SCT (Fig 2) illustrate the need to perform fetal MR imaging to establish an accurate diagnosis.

This patient was referred for further investigation of a sacrococcygeal mass. A 30-week sonography showed an external cystic mass with internal septation that measured $8.9 \times 7.7 \times 9.5 \mathrm{~cm}($ Fig $2 A,-B)$. MR imaging at 30 weeks revealed pelvic extension previously undetected on sonography (Fig $2 C,-D$ ) and thus confirmed the diagnosis of type 2 SCT. A follow-up sonography at 34 weeks showed that the external cystic component had increased to $12 \times 9.4 \times 8 \mathrm{~cm}$. No other fetal anomalies were detected. Amniotic fluid AFP and acetylcholinesterase levels were normal. The AFI was also within normal limits. The increase in size of the mass between weeks 30 and 34 raised the risk of both rupture and preterm delivery; thus, cesarean delivery was scheduled for 34 weeks and 2 days.

The cesarean delivery was uncomplicated. Infant Apgar scores were 8 and 9 at 1 and 5 minutes, respectively. The infant was polycythemic (hematocrit level of 62). SCT was confirmed at delivery and surgically treated.

\section{Discussion}

Although cystic SCT and terminal myelocystocele may have a similar appearance on prenatal sonography, they differ substantially in associated perinatal risk and long-term morbidity. In addition, appropriate surgical correction is performed by pediatric surgery for the former and pediatric neurosurgery for the latter.

SCT is the most common congenital tumor, occurring 1 in 40,000 live births, with a female predisposition (approximately 3:1). SCT may be subclassified as follows: Type 1 is predominantly external with a minimal presacral component; type 2 is also exophytic with significant intrapelvic extension. Type 3 has an external mass with a predominant intrapelvic mass, and type 4 is entirely presacral. ${ }^{2}$ Most SCTs are solid or solid-cystic, with $15 \%$ being purely cystic. ${ }^{3}$ SCT is not a neural tube defect, and there is typically no spinal dysraphism, 

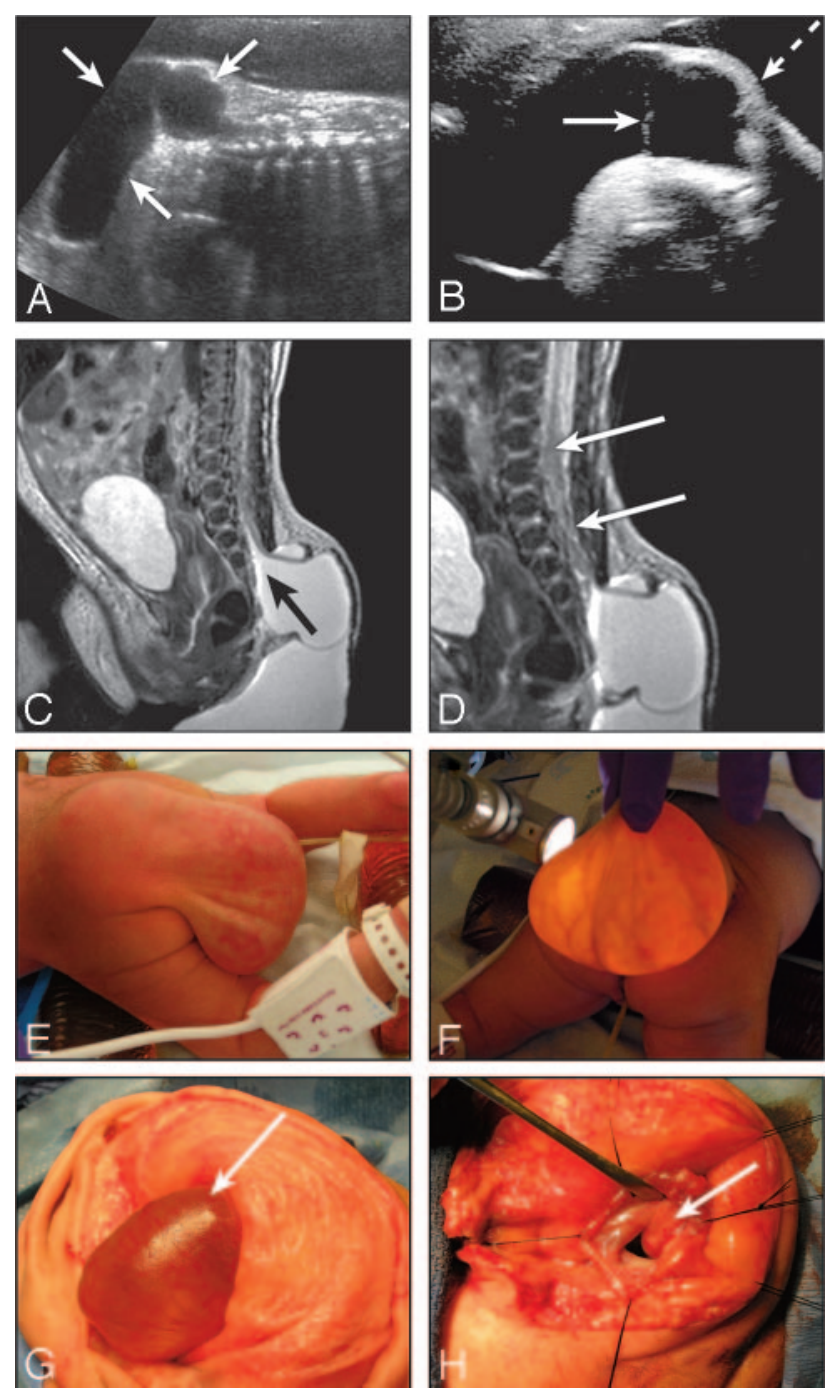

Fig 1. Terminal myelocystocele

A, 30-week fetal sonography, sagittal spine view, shows a large cystic mass (arrows) arising from distal tip of sacrum.

$B$, Axial view shows a single internal septation (arrow). The mass is contiguous with skin (dashed arrow).

C, T2-weighted neonatal spine MR image shows that the cystic mass communicates with the spinal canal (arrow).

$D$, Same view as $C$ shows that cord is distended, dysmorphic, and tethered to the mass (arrows).

$E$ and $F$, Preoperative image of the terminal myelocystocele $(E)$, with transillumination $(F)$. $G$, Intraoperative view shows terminal dilation of central canal (arrow) - septation seen on sonography was this cyst wall.

$H$, Intraoperative view shows spinal canal and tethered cord (arrow).

though there may be occasional sacral dysgenesis or hemivertebrae. ${ }^{4}$

Neonates with SCT have an excellent prognosis depending on surgical resection and malignant potential. However, the fetus is at high risk for perinatal complications and death. Complications include tumor rupture and hemorrhage, preterm labor, dystocia, and increased risk of polyhydramnios or oligohydramnios. Solid SCTs are associated with increased AFP levels and risk of high-output cardiac failure and hydrops. ${ }^{5}$ To minimize risk of rupture, Gross et $\mathrm{al}^{5}$ recommend cesarean delivery for fetuses with SCTs of more than $5 \mathrm{~cm}$ in diameter.
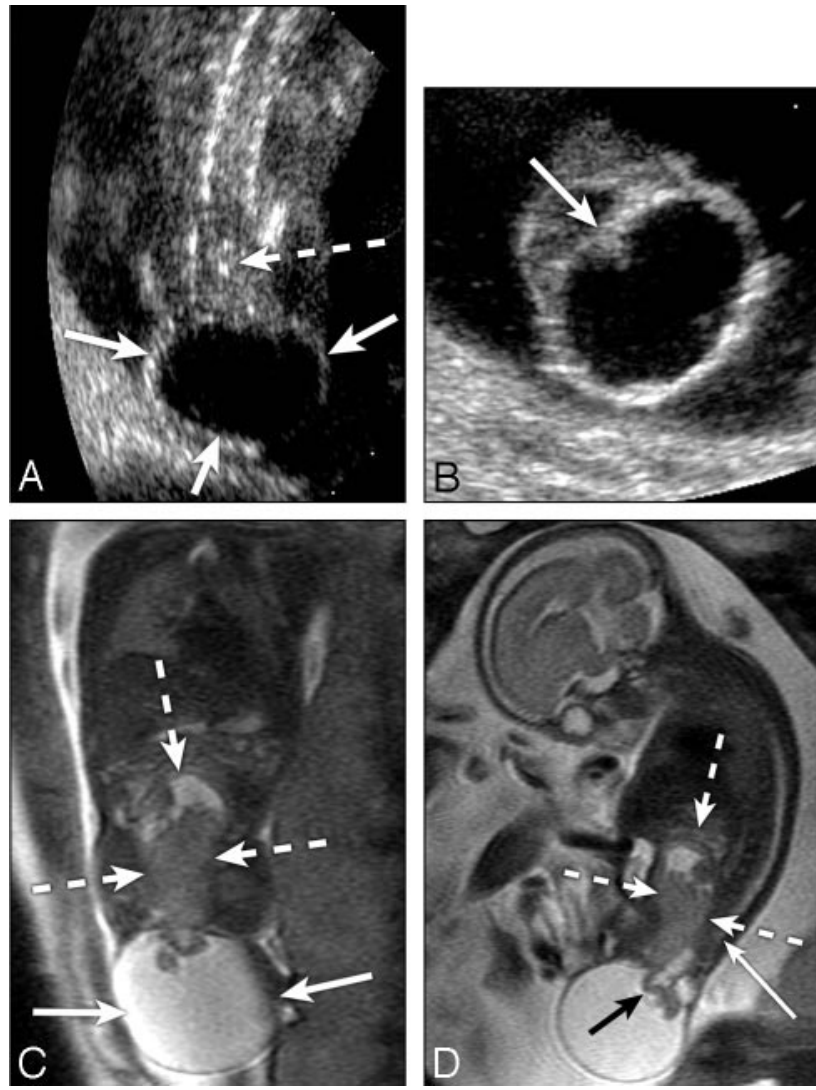

Fig 2. Type 2 SCT in a 28-week fetus.

$A$, Coronal sonography view shows cystic mass (arrows) arising from the tip of the sacrum (dashed arrow).

$B$, Axial view shows internal septation (arrow)

C, T2-weighted fetal MR image, coronal view, shows a cystic mass (arrows) and a significant intracorporeal solid component (dashed arrows).

$D$, T2-weighted fetal MR image, sagittal view, confirms intracorporeal extension (dashed arrows). The internal septation (black arrow) seen by sonography is part of the mass, not an extension of the spinal canal (white arrow points to tip of high-signal-intensity CSF in spinal canal).

Terminal myelocystocele is a skin-covered neural tube defect: a localized dilation of the spinal cord central canal herniates through a dorsal spinal defect, creating an ependymalined sac. Terminal myelocystoceles constitute approximately $4 \%-6.5 \%$ of skin-covered lower spine masses and also occur more frequently in females. ${ }^{1,6,7}$ They are often associated with omphalocele, extrophy of the bladder, imperforate anus, and sacral agenesis (OEIS complex), though they also occur in isolation, as our case demonstrates. The presence of abdominal wall defects is associated with higher risk of neurologic deficits. ${ }^{6}$

In contrast to SCT, terminal myelocystocele is associated with spinal dysraphism and AFP levels are typically normal. Prenatal complications are rare and vaginal deliveries are often considered safe. ${ }^{8}$ In general, perinatal morbidity and mortality are much lower in terminal myelocystocele than in SCT, though measured long-term outcomes are more variable.

The differential diagnosis for perinatal sacrococcygeal masses is vast, including meningocele, myelomeningocele, myelocystocele, teratoma, lipoma, hamartoma, lymphangioma, hemangioma, chordoma, and ependymoma. Although case reports have identified misdiagnosis of SCT as myelomeningocele, myelocystocele is typically not listed in the dif- 
ferential diagnosis of SCT. ${ }^{2,9}$ The key imaging distinction between these cystic masses is the dysraphic spine and the continuity of the protruding sac, with the spinal cord central canal associated with terminal myelocystocele. At the sacrococcygeal level, dysraphism is difficult to visualize by prenatal sonography. In our index case, the association between acutely progressive polyhydramnios and elevated AFP levels led to a presumptive prenatal diagnosis of SCT. Ultrafast fetal MR imaging may show the relationships of a mass to the sacrum and spinal cord and better demonstrate the solid component of mixed SCT than sonography. Fetal MR imaging may thus be a useful adjunct in the differential diagnosis of cystic lesions of the sacrococcygeal spine. ${ }^{10}$ In addition, it was only when post-natal MR imaging demonstrated terminal myelocystocele that pediatric neurosurgery became involved in the case. Accurate prenatal diagnosis may be useful in prospective perinatal management as well as parental counseling.

\section{Acknowledgments}

We thank Shirley McCartney, PhD, and Andy Rekito for editorial assistance.

\section{References}

1. McLone DG, Naidich TP. Terminal myelocystocele. Neurosurgery 1985;16:36-43

2. Altman RP, Randolph JG, Lilly JR. Sacrococcygeal teratoma: American Academy of Pediatrics Surgical Section Survey-1973. J Pediatr Surg 1974;9:389-98

3. Ein SH, Adeyemi SD, Mancer K. Benign sacrococcygeal teratomas in infants and children: a 25 year review. Ann Surg 1980;191:382-84

4. Guvenc BH, Etus V, Muezzinoglu B. Lumbar teratoma presenting intradural and extramedullary extension in a neonate. Spine J 2006;6:90-93

5. Gross SJ, Benzie RJ, Sermer M, et al. Sacrococcygeal teratoma: prenatal diagnosis and management. Am J Obstet Gynecol 1987;156:393-96

6. James HE, Lubinsky G. Terminal myelocystocele. J Neurosurg 2005;103:443-45

7. Gupta DK, Mahapatra AK. Terminal myelocystoceles: a series of 17 cases. J Neurosurg 2005;103:344-52

8. Luthy DA, Wardinsky T, Shurtleff DB, et al. Cesarean section before the onset of labor and subsequent motor function in infants with meningomyelocele diagnosed antenatally. N Engl J Med 1991;324:662-66

9. Rumack C, Wilson S, Charboneau J, eds. Diagnostic Ultrasound. 3rd ed. St. Louis: Elsevier Mosby; 2005:1297-301

10. Simon EM. MRI of the fetal spine. Pediar Radiol 2004;34:712-19 